\title{
Is mitochondrial DNA depletion involved in Alzheimer's disease?
}

\author{
Benjamín Rodríguez-Santiago ${ }^{1}$, Jordi Casademont ${ }^{2}$ and Virginia Nunes ${ }^{*, 1}$
}

\author{
${ }^{1}$ Medical and Molecular Genetics Center, Institut de Recerca Oncologica, Barcelona, Spain; ${ }^{2}$ Muscle Research \\ Group, Hospital Clínic, IDIBAPS, Universitat de Barcelona, Barcelona, Spain
}

\begin{abstract}
Several studies have suggested that mitochondrial metabolism disturbances and mitochondrial DNA (mtDNA) abnormalities may contribute to the progression of the pathology of Alzheimer's disease (AD). In this study we have investigated whether the amount of mtDNA is modified in different brain regions (cerebellum, hippocampus and frontal cortex) of confirmed AD necropsies and in blood of living AD patients. We used a real-time PCR method to analyse the mtDNA relative abundance in brain regions from 12 AD and seven controls and from a group of blood samples (17 living AD patients and 11 controls). MtDNA from blood samples together with hippocampus and cerebellum brain areas did not show differences between controls and AD. However, AD patients showed a $28 \%$ decrease in the amount of mtDNA in the frontal cortex when compared to controls for this specific area. Since frontal cortex is a severely affected region in $A D$, our results support the hypothesis that mitochondrial defects may play a role in the pathogenesis of AD. European Journal of Human Genetics (2001) 9, 279-285.
\end{abstract}

Keywords: mtDNA; depletion; mitochondria; Alzheimer's disease; brain

\section{Introduction}

Alzheimer's disease (AD) is one of the major causes of dementia. It is an age-related condition affecting $11 \%$ of the population over 65 years of age and $50 \%$ over age 85 . AD is best characterised as a chronic brain disorder, having a lengthy pre-clinical phase followed by a malignant stage associated with neuronal degeneration, the loss of specific synaptic connections and the progressive erosion of higher cognitive functions. ${ }^{1}$ AD has two major neuropathological hallmarks: an abundance of extracellular amyloid deposits, known as senile plaques, and fibrillar protein deposits within neurons, known as neurofibrillary tangles. ${ }^{2}$

The fundamental reason for the degeneration of nerve cells and synaptic connections that underlies the emergence of dementia has been elusive. It is widely considered that genetic factors, acting independently or in concert with other genetic and/or environmental factors, modify the risk

*Correspondence: V Nunes, Centre de Genètica Mèdica i Molecular-IRO, Hospital Duran i Reynals, Autovía de Castelldefels km 2.7, 08907 L'Hospitalet del Llobregat, Barcelona, Spain.

Tel: +34 932607775; Fax: +34 932607776; E-mail: vnunes@iro.es Received 25 September 2000; revised 9 December 2000; accepted 2 January 2001 of developing the disease. ${ }^{3}$ Many genes associated with the disease have been identified. Some of these genes are involved in early onset forms of the disease and have a direct causal effect: The amyloid precursor protein (APP) gene located on chromosome 21 and presenilin genes 1 and 2 located on chromosome 14 and 1 respectively. The apolipoprotein E (apo E) gene (located on chromosome 19), the $\alpha_{2^{-}}$ macroglobulin gene located on chromosome 12, and other unidentified genes may determine susceptibility in late-onset forms and sporadic cases. ${ }^{4}$

On the other hand, several studies have suggested that mitochondrial dysfunction may contribute to the progression of AD. Some evidence includes defects in the electron transport chain, ${ }^{5}$ decreased cytochrome oxidase activity, ${ }^{6}$ impairment of mitochondrial oxidative metabolism, ${ }^{7}$ mtDNA point mutations, ${ }^{8,9}$ rearrangements and deletions, ${ }^{10,11}$ and decreased expression of genes. ${ }^{12,13}$ Such defects could interfere with other features in which mitochondria are involved: Calcium homeostasis, ${ }^{14}$ apoptotic pathways, ${ }^{15,16}$ reactive oxygen species (ROS) generation and amyloid metabolism, ${ }^{16}$ leading to a number of deleterious consequences. These features could form a vicious circle, with further impairment of the function of the respiratory machinery which, in turn, promotes the generation of more 
free radicals, oxidative damage to membrane phospholipids and proteins, and more mtDNA mutations in a sort of catastrophic vicious cycle, leading to an increased neuronal susceptibility to cell death.

The reduction in mtDNA copy number (mtDNA depletion) has been described in several pediatric syndromes, ${ }^{17-19}$ and in some toxic situations, ${ }^{20,21}$ with an expanding spectrum of clinical presentations, including central nervous system involvement. $^{22}$ To our knowledge, mtDNA depletion has not been tested in neurodegenerative disorders, and specifically in $\mathrm{AD}$, perhaps because up to now, the diagnosis of mtDNA depletion diseases has been based only on Southern blot analyses hybridising simultaneously with mtDNA and r18S probes. ${ }^{17,22-24}$ Such a technique requires a relatively high amount of DNA, is difficult to standardise and is susceptible to misinterpretations. In the present work we have used a real time PCR quantification method based on Lightcycler technology (Roche Molecular Biochemicals, Germany) to investigate mtDNA in blood samples of 17 Alzheimer patients and 11 controls and in post-mortem samples from different brain regions of 12 Alzheimer patients and seven controls. Additionally, and although mtDNA depletion in blood has not been described in any disease, some authors have reported cytochrome oxidase deficiency ${ }^{25}$ and oxidative damage to mtDNA in lymphocytes from AD patients. ${ }^{26}$ Therefore, we have also performed the analysis in blood in order to look for possible defects in the levels of mtDNA in living AD patients.

\section{Materials and methods}

\section{Subjects}

Brain samples of cerebellum, hippocampus and frontal cortex from 12 histopathologically confirmed AD patients and from seven controls were obtained from the Neurologic Tissue Bank of the Hospital Clínic-Universitat de Barcelona. Areas finally analysed from each subject are summarised in Table 1. In all cases the time between death and frozen storage of neurological tissues was inferior to $12 \mathrm{~h}$. The age range was 56-84 years for $\mathrm{AD}$ and 55-70 years for controls. Control

Table 1 MtND2/18S ratios and statistical data in blood and in the different brain areas

\begin{tabular}{|c|c|c|c|c|c|c|c|}
\hline Subject & Age & $\begin{array}{r}\text { Brain } \\
\text { Cerebellum }\end{array}$ & $\begin{array}{c}\text { MtND2/r18S ratic } \\
\text { Hippocampus }\end{array}$ & Cortex & \multicolumn{3}{|c|}{ Blood } \\
\hline \multicolumn{8}{|c|}{ Controls } \\
\hline 1 & 69 & 0.29 & 2.63 & 3.00 & 11 & 66 & 0.69 \\
\hline 2 & 69 & 0.36 & 0.63 & 4.52 & 12 & 76 & 0.29 \\
\hline 3 & 67 & 0.48 & 6.06 & 7.58 & 13 & 82 & 0.13 \\
\hline 4 & 63 & 0.75 & 2.66 & 3.87 & 14 & 79 & 0.07 \\
\hline 5 & 55 & 0.72 & & 2.49 & 15 & 74 & 0.02 \\
\hline 6 & 66 & 0.75 & & 5.63 & 16 & 78 & 0.03 \\
\hline \multirow[t]{5}{*}{7} & 70 & 0.54 & & 4.46 & 17 & 80 & 0.15 \\
\hline & & & & & 18 & 77 & 0.04 \\
\hline & & & & & 19 & 67 & 0.11 \\
\hline & & & & & 20 & 61 & 0.06 \\
\hline & & & & & 21 & 76 & 0.06 \\
\hline Mean & 65.57 & 0.56 & 3.00 & 4.51 & & 73.90 & 0.16 \\
\hline SD & 5.22 & 0.19 & 2.25 & 1.71 & & 6.63 & 0.20 \\
\hline \multicolumn{8}{|l|}{ Patients } \\
\hline 1 & 73 & 0.41 & 1.82 & 3.23 & 13 & 67 & 0.10 \\
\hline 2 & 68 & 0.51 & 2.38 & 2.13 & 14 & 71 & 0.37 \\
\hline 3 & 79 & 0.54 & 1.58 & 1.57 & 15 & 73 & 0.17 \\
\hline 4 & 78 & 0.64 & 4.21 & 4.97 & 16 & 87 & 0.42 \\
\hline 5 & 56 & 0.73 & 1.04 & 2.48 & 17 & 69 & 0.74 \\
\hline 6 & 76 & 0.75 & 6.06 & 8.00 & 18 & 84 & 0.11 \\
\hline 7 & 64 & 0.34 & 1.31 & 1.60 & 19 & 83 & 0.32 \\
\hline 8 & 80 & 0.43 & 2.15 & 4.94 & 20 & 59 & 0.38 \\
\hline 9 & 62 & 0.54 & 3.46 & 4.31 & 21 & 70 & 0.17 \\
\hline 10 & 84 & 0.53 & 1.16 & 1.91 & 22 & 76 & 0.14 \\
\hline 11 & 82 & 0.24 & & 2.61 & 23 & 87 & 0.04 \\
\hline \multirow[t]{6}{*}{12} & 66 & 0.59 & & 1.24 & 24 & 79 & 0.09 \\
\hline & & & & & 25 & 83 & 0.09 \\
\hline & & & & & 26 & 82 & 0.04 \\
\hline & & & & & 27 & 78 & 0.03 \\
\hline & & & & & 28 & 69 & 0.03 \\
\hline & & & & & 29 & 71 & 0.05 \\
\hline Mean & 72.33 & 0.54 & 2.61 & 3.25 & & 75.36 & 0.20 \\
\hline SD & 8.95 & 0.15 & 1.67 & 1.98 & & 7.96 & 0.19 \\
\hline
\end{tabular}

Each ratio results from the mean of two experiments (typical replicate CV was 1-12\%) for each sample. (SD: standard deviation). 
samples belonged to individuals without dementia or other neurological disease in whom the neuropathological analyses excluded the presence of senile plaques and neurofibrillary tangles.

Blood samples of 17 living AD patients (age range: 59-87) diagnosed by standard neurological criteria ${ }^{27}$ and from 11 age-matched controls (age range: 61-80) were also analysed.

All AD patients were sporadic cases. The subjects' consent was obtained according to the declaration of Helsinki and following the rules of the Ethical Committee of the Centre.

\section{DNA analyses}

Total DNA was prepared from $50 \mathrm{mg}$ of brain necropsy tissue and from $20 \mathrm{ml}$ of blood following standard procedures based on phenol-chloroform extraction. A rapid and sensitive PCR real time method was used for quantification of mtDNA and nuclear DNA (represented by the ribosomal 18 genes). The method used a double-stranded DNA dye (SYBR Green I) to continuously monitor product formation and was able to quantify samples ranging up to $4 \log$ units in concentration. The sensitivity of SYBR Green I detection is limited by non-specific product formation, which is monitored by fluorescence acquisition at temperatures at which only specific products are double-stranded. ${ }^{28}$ The values found in each sample for the two genes (representative of mtDNA and nuclear DNA, respectively) allowed the calculation of a ratio mtDNA/nDNA to make comparisons between the different groups.

\section{Measurement of mtND2 and $\mathrm{r} 18 \mathrm{~S}$ sequences by continuous monitoring of PCR}

Real time PCR was performed in a fluorescence temperature cycler Lightcycler (Roche Molecular Biochemicals, Germany). Amplifications were carried out in a 20- $\mu$ l final volume containing $3 \mathrm{mM} \mathrm{MgCl}_{2}$ for mtND2 or $2 \mathrm{~mm}$ for r18S, $0.5 \mu \mathrm{M}$ of each primer and $2 \mu \mathrm{l}$ of the LightcyclerFastStart DNA Master SYBR Green I mix. The oligonucleotide primers used to detect the mtND2 sequences were L456 (5'-GCCCTAGAAATAAACATGCTA-3') and H479 (5'GGGCTATTCCTAGTTTTATT-3'). The oligonucleotide primers used to detect the r18S sequences were 18SFOR ( $5^{\prime}$ ACGGACCAGAGCGAAAGCAT-3') and 18SREV (5'-GGACATCTAAGGGCATCACAGAC-3'). The mtND2 PCR program included: initial denaturation at $95^{\circ} \mathrm{C}$ for $10 \mathrm{~min}$ followed by 40 amplification cycles consisting of heating at $20^{\circ} \mathrm{C} / \mathrm{s}$ to $94^{\circ} \mathrm{C}$ with a 1 -s hold, annealing at $20^{\circ} \mathrm{C} / \mathrm{s}$ to $53^{\circ} \mathrm{C}$ with a 10 -s hold and extension at $20^{\circ} \mathrm{C} / \mathrm{s}$ to $72^{\circ} \mathrm{C}$ with a 10 -s hold. The fluorescent product was detected at the last step of each cycle by single acquisition. After amplification, a melting curve was acquired by heating the product at $20^{\circ} \mathrm{C} / \mathrm{s}$ to $95^{\circ} \mathrm{C}$, cooling at $20^{\circ} \mathrm{C} / \mathrm{s}$ to $65^{\circ} \mathrm{C}$, and slowly heating it at $0.2^{\circ} \mathrm{C} / \mathrm{s}$ to $94^{\circ} \mathrm{C}$ with continuous fluorescence collection. Melting curves were used to determine the specificity of the PCR products. $^{29}$ The r18S PCR program was performed in the same conditions as the ND2 program, except for: the annealing temperature $\left(65^{\circ} \mathrm{C}\right)$, one melting curve acquisition step (cooling at $20^{\circ} \mathrm{C} / \mathrm{s}$ to $72^{\circ} \mathrm{C}$ ), and the number of cycles (45 in the r18S PCR program). Variations in sample loading or due to PCR tube-to-tube efficiency were corrected by performing two replicates for each standard and unknown sample.

\section{Standard design}

A known amount of human DNA (initial concentration of $15 \mathrm{ng} / \mu \mathrm{l}$ ) from the Lightcycler control Kit was serially diluted and amplified for the mtND2 and the r18S regions to obtain two standard log-linear regression lines. The Lightcycler software first normalises each sample by background substraction. Then, a fluorescence threshold ( $\cong 5 \%$ of full scale) is used to determine fractional cycle numbers that correlate inversely to the $\log$ of the initial template concentration. The least-squares best fit of the standards is used to calculate the amount of template initially present in the samples. Typical standard curves obtained for mtND2 and r18S are represented in Figure 1A,B.

\section{Statistical analysis}

The mean value of the two replicates (typical replicate coefficients of variation (CV) were $1-12 \%$ ) for each sample was used to determine the values of mtDNA and r18S amount. The mtND2/18S mean ratios of each group were used for comparisons between them. An unpaired $t$-test previous verification that the samples had the same variance (Levene) was used to compare controls and $\mathrm{AD}$ patients.

\section{Results}

Amplification of mtND2 and r18S derived from amplifying fourfold dilutions of standard DNA provided two log-linear control standard lines $\left(r^{2}=0.99\right.$ for both cases, see Figure 1C,D). DNA samples were amplified following the same standard conditions by continuously monitored PCR. The reason for choosing mtDNA primers in the ND2 gene was to avoid the common mtDNA deletion region. Furthermore, the ND2 gene is close to the light chain origin of replication (which is an area usually not involved in mtDNA deletions) and no nuclear pseudogene has been described in this region. The mtDNA/r18S quantification is found by determining the cycle number at which the product fluorescence becomes greater than a defined threshold. The method is based on the premise that the greater the quantity of initial DNA template in a sample, the fewer the PCR cycles required to reach this threshold, ${ }^{28}$ as it can be seen in Figure 1. Also, the results of the two replicates of standard samples can be observed. The specificity of the products was assessed by monitoring the melting curve acquisition (Figure 2).

Since the quantification of the r18S housekeeping gene is used to standardise the results, the $\mathrm{mtND} 2 / \mathrm{r} 18 \mathrm{~S}$ ratio can be used to standardise the relative mtDNA abundance in each sample such that the relative mtDNA contents of various 


\section{mtND2 profiles}

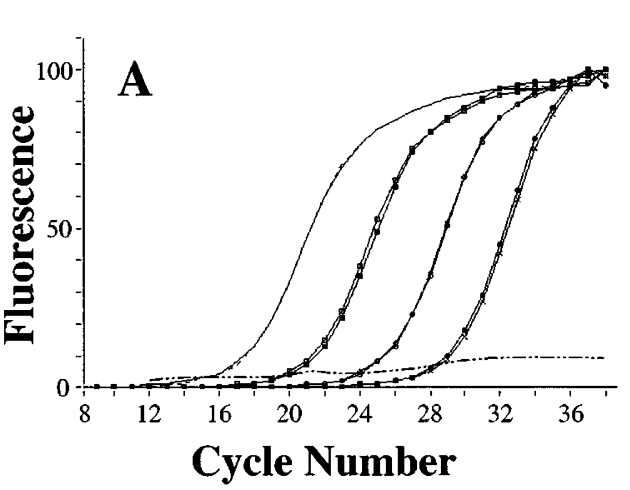

C

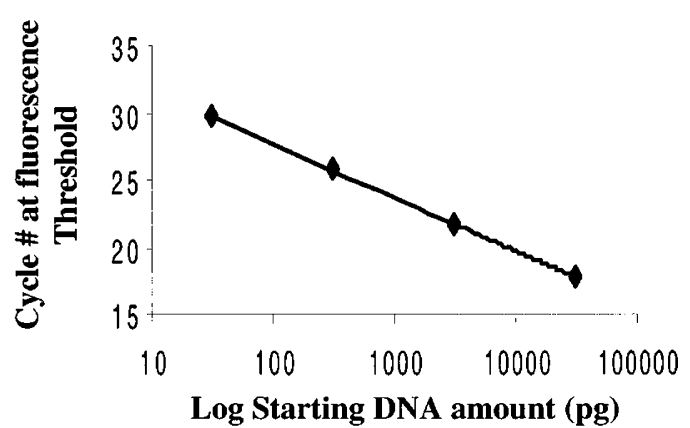

r18S profiles
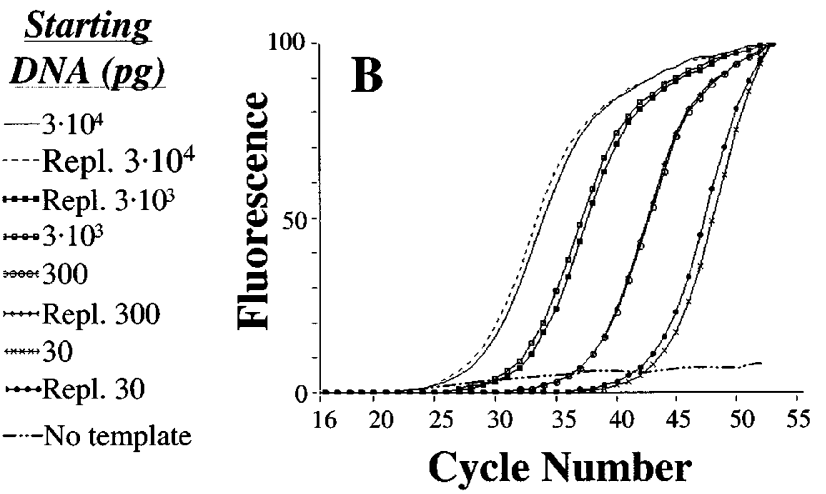

D

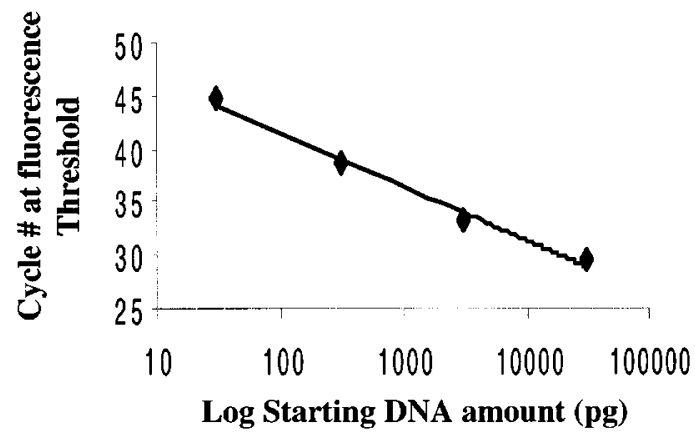

Figure 1 Fluorescent-amplification profiles and linear regression lines for mtND2 and r18S sequences. A, B Background-subtracted fluorescent emission of samples plotted as a function of cycle number. Serially dilluted samples of known DNA template were amplified for A mtND2 and B r18S sequences as described in Materials and methods. C, D These panels depict the fluorescence threshold for the samples presented in A and B plotted as a function of the starting DNA amount. The solid line indicates a linear regression fit to the data.

samples can be compared. The mtND2/r18S ratios calculated as the mean of two experiments for each sample are compiled in Table 1 . When the groups were compared it could be observed that patients and controls presented similar amounts of mtDNA in cerebellum and hippocampus brain regions and in blood (Figure 3 and Table 1). On the other hand, $\mathrm{AD}$ patients showed a $28 \%$ reduction of their mtDNA amount in frontal cortex, although this difference did not reach statistical significance $(P=0.18)$. No presence of large rearrangements were observed by Southern blot in any sample (data not shown).

\section{Discussion}

Although Alzheimer's disease is no longer the condition described in texts written some 10 years ago the aetiology of the disease remains enigmatic. The pathogenic pathways involved in the development of brain dysfunction in AD are much debated in the literature. Particularly, involvement of mitochondrial dysfunction remains controversial. Whereas it is clear that a primary mtDNA defect is not the substrate of sporadic cases of $\mathrm{AD}$, the eventual contribution of mitochondrial dysfunction to worsen the clinical features is a possibility that can not be ruled out.

The objective of the present study was to assess whether mtDNA depletion, one increasingly recognised condition among the various molecular defects found in mitochondrial disorders, could be present in AD. Such studies can now rely on real-time quantification PCR technology which eludes a series of methodological and interpretational problems presented by the classical Southern blot analysis. In this way, the rapidity, reproducibility and specially the sensitivity of this PCR method makes it the technique of choice for detecting mtDNA depletion.

Although no depletion of mtDNA in lymphocytes has ever been described, previous studies revealing abnormalities in lymphocytes from $\mathrm{AD}$ patients ${ }^{25,26}$ and the convenience of finding a parameter that could easily discriminate individuals prompted us to analyse the levels of mtDNA from lymphocytes of living patients. However, the results obtained 
$\mathbf{A}$

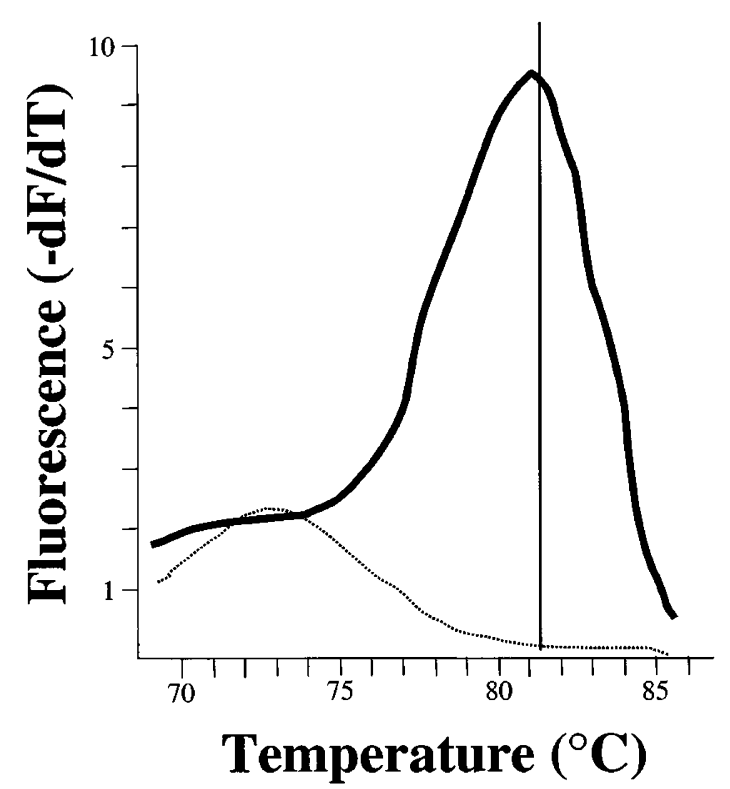

B

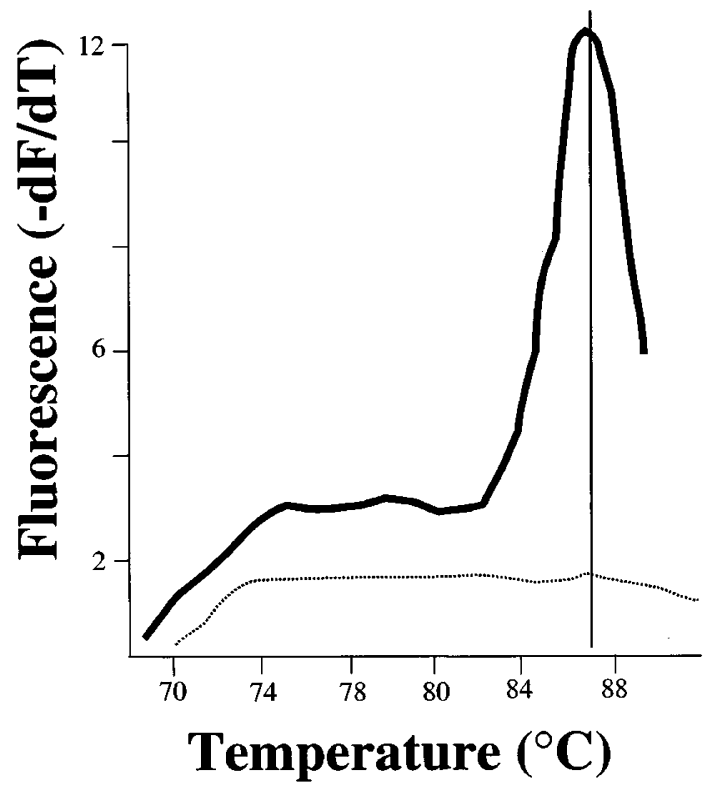

Figure 2 Melting curves for mtND2 and r18S. A mtND2 and B r18S PCR end products for samples containing DNA (solid line) or without template (light line). Following amplifications, dsDNA fluorescence was measured as the temperature was increased at $0.2^{\circ} \mathrm{C} / \mathrm{s}$ from 65 (mtND2) or $72(\mathrm{r} 18 \mathrm{~S})$ to $94^{\circ} \mathrm{C}$. The rate of fluorescence change with changing temperature (-dF/dT) was plotted as a function of temperature. The mtND2 product $T_{m}$ was $81.5^{\circ} \mathrm{C}$ and for $r 18 \mathrm{~S}$ was $87^{\circ} \mathrm{C}$.

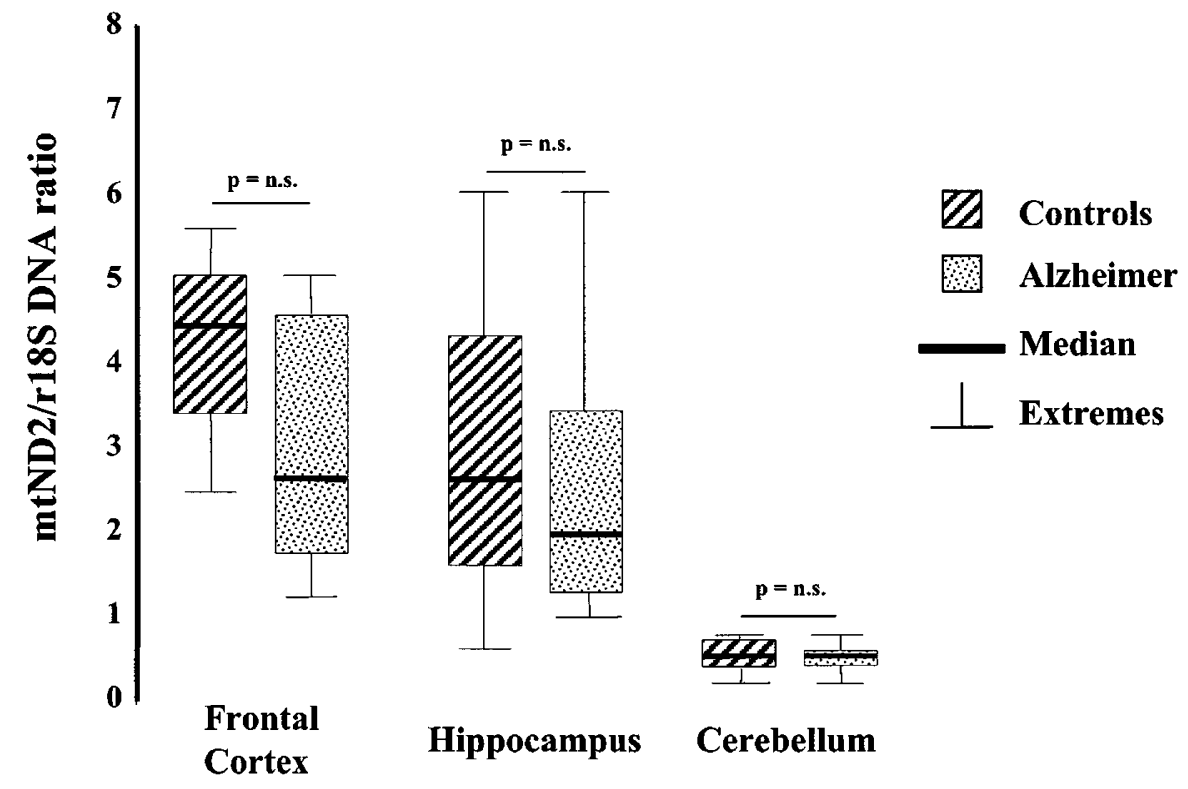

Figure 3 Comparison of Alzheimer and control mtND2/r18S ratios in the different brain areas analysed. Boxplots of the mitochondrial (mtND2)/nuclear (r18S) DNA ratio in the regions of the brain analysed. The numerical data for ages, medians and standard deviation values are presented in Table 1. (n.s: not significant).

indicate that there were no differences between $\mathrm{AD}$ patients and matched controls, so this parameter cannot be used for developing a diagnostic test for AD.
In the present study we have found a $28 \%$ reduction in mtDNA content in frontal cortex from patients with AD. Although statistically not significant, the data might suggest 
mtDNA decrease in the frontal cortex of some patients. Mitochondrial studies in brain have to consider the differences in the relative abundance of mtDNA contents depending on the brain area analysed. Furthermore, it has to be noted that $\mathrm{AD}$ is a disease characterised by a very marked differential severity of the neuronal damage depending on the cortex area examined, as other authors have reported. ${ }^{10}$ On the other hand, an age-related increase of mtDNA amount has been found in the frontal cortex of elderly individuals. ${ }^{30}$ This increase in mtDNA content with age may be considered a gene dosage compensatory mechanism for mitochondria with altered capacity for substrate oxidation.

Once taken into account such considerations, the reduction of mtDNA circumscribed to frontal cortex in our group of $\mathrm{AD}$ patients is in accordance with the $\mathrm{AD}$ neuronal loss preferentially occurring in frontal cortex and less in cerebellum or hippocampus. The physiological increase in mtDNA of frontal cortex in normal individuals is completely overcomed in $\mathrm{AD}$ patients. This mtDNA defect seems to have, therefore, an anatomic specificity, and may contribute to impaired energy generation in $\mathrm{AD}$ reported by other authors.

The real contribution of such mtDNA defect in the pathophysiology of $\mathrm{AD}$ remains elusive. The precise balance between nuclear and mitochondrial genome, steady-state levels of mitochondrial RNA, mitochondrial respiratory chain function, calcium homeostasis, ROS production, amyloid metabolism, and cell signalling may provide the information necessary to complete our understanding of the mitochondrial role in neuronal loss in such devastating disease.

\section{Acknowledgements}

This work was supported by grants Fundació La Marató de TV3 2002/ 97 and 2102/97 and CICYT SAF 99-0079. B Rodríguez-Santiago is supported by Fundació La Marató de TV3. V Nunes is supported by Institut Català de la Salut. We thank the Neurologic Tissue Bank (Serveis Cientifico-Tècnics, Universitat de Barcelona) for providing the samples, Montse Gómez Zaera for comments on the manuscript, Carlos Manchado for technical support and Helena Kruyer for editorial help.

\section{References}

1 Vickers JC, Dickson TC, Adlard PA, Saunders HL, King CE, McCormack G: The cause of neuronal degeneration in Alzheimer's disease. Prog Neurobiol 2000; 60: 139-165.

2 Dickson DW: The pathogenesis of senile plaques. J Neuropathol Exp Neurol 1997; 56: $321-339$.

3 Ray WJ, Ashall F, Goate AM: Molecular pathogenesis of sporadic and familial forms of Alzheimer's disease. Mol Med Today 1998; 4: $151-157$.

4 Christen Y: Oxidative stress and Alzheimer disease. Am J Clin Nutr 2000; 71: 621S-629S.

5 Parker Jr WD, Parks J, Filley CM, Kleinschmidt-DeMasters BK: Electron transport chain defects in Alzheimer's disease brain [see comments]. Neurology 1994; 44: 1090-1096.
6 Mutisya EM, Bowling AC, Beal MF: Cortical cytochrome oxidase activity is reduced in Alzheimer's disease. J Neurochem 1994; 63 : 2179-2184.

7 Redjems-Bennani N, Jeandel C, Lefebvre E, Blain H, Vidailhet M, Gueant JL: Abnormal substrate levels that depend upon mitochondrial function in cerebrospinal fluid from Alzheimer patients. Gerontology 1998; 44: 300-304.

8 Egensperger R, Kosel S, Schnopp NM, Mehraein P, Graeber MB: Association of the mitochondrial tRNA(A4336G) mutation with Alzheimer's and Parkinson's diseases. Neuropathol Appl Neurobiol 1997; 23: 315-321.

9 Hutchin T, Cortopassi G: A mitochondrial DNA clone is associated with increased risk for Alzheimer disease. Proc Natl Acad Sci USA 1995; 92: 6892-6895.

10 Corral-Debrinski M, Horton T, Lott MT et al: Marked changes in mitochondrial DNA deletion levels in Alzheimer brains. Genomics 1994; 23: 471-476.

11 Hamblet NS, Castora FJ: Elevated levels of the Kearns-Sayre syndrome mitochondrial DNA deletion in temporal cortex of Alzheimer's patients. Mutat Res 1997; 379: 253-262.

12 Aksenov MY, Tucker HM, Nair P et al: The expression of several mitochondrial and nuclear genes encoding the subunits of electron transport chain enzyme complexes, cytochrome c oxidase, and NADH dehydrogenase, in different brain regions in Alzheimer's disease. Neurochem Res 1999; 24: 767 - 774.

13 Chandrasekaran K, Hatanpaa K, Rapoport SI, Brady DR: Decreased expression of nuclear and mitochondrial DNAencoded genes of oxidative phosphorylation in association neocortex in Alzheimer disease. Brain Res Mol Brain Res 1997; 44 $99-104$.

14 Sheehan JP, Swerdlow RH, Miller SW et al: Calcium homeostasis and reactive oxygen species production in cells transformed by mitochondria from individuals with sporadic Alzheimer's disease. J Neurosci 1997; 17: 4612-4622.

15 Suzuki A, Tsutomi Y, Yamamoto N, Shibutani T, Akahane K: Mitochondrial regulation of cell death: mitochondria are essential for procaspase 3-p21 complex formation to resist Fasmediated cell death. Mol Cell Biol 1999; 19: 3842-3847.

16 Deng G, Su JH, Ivins KJ, Van Houten B, Cotman CW: Bcl-2 facilitates recovery from DNA damage after oxidative stress. Exp Neurol 1999; 159: 309-318.

17 Moraes CT, Shanske S, Tritschler HJ et al: mtDNA depletion with variable tissue expression: a novel genetic abnormality in mitochondrial diseases. Am J Hum Genet 1991; 48: 492-501.

18 Ricci E, Moraes CT, Servidei S, Tonali P, Bonilla E, DiMauro S: Disorders associated with depletion of mitochondrial DNA. Brain Pathol 1992; 2: 141-147.

19 Poulton J, Sewry C, Potter CG et al: Variation in mitochondrial DNA levels in muscle from normal controls. Is depletion of mtDNA in patients with mitochondrial myopathy a distinct clinical syndrome. J Inherit Metab Dis 1995; 18: 4-20.

20 Casademont J, Barrientos A, Grau JM et al: The effect of zidovudine on skeletal muscle mtDNA in HIV-1 infected patients with mild or no muscle dysfunction. Brain 1996; 119: $1357-1364$

21 Dalakas MC, Illa I, Pezeshkpour GH, Laukaitis JP, Cohen B, Griffin JL: Mitochondrial myopathy caused by long-term zidovudine therapy [see comments]. N Engl J Med 1990; 322: $1098-1105$.

$22 \mathrm{Vu} \mathrm{TH}$, Sciacco M, Tanji K et al: Clinical manifestations of mitochondrial DNA depletion. Neurology 1998; 50: 1783-1790.

23 Arnaudo E, Dalakas M, Shanske S, Moraes CT, DiMauro S, Schon EA: Depletion of muscle mitochondrial DNA in AIDS patients with zidovudine-induced myopathy. Lancet 1991; 337: $508-$ 510 .

24 Campos Y, Martin MA, Garcia-Silva T et al: Clinical heterogeneity associated with mitochondrial DNA depletion in muscle. Neuromuscul Disord 1998; 8: 568-573.

25 Parker Jr WD: Cytochrome oxidase deficiency in Alzheimer's disease. Ann NY Acad Sci 1991; 640: 59-64. 
26 Mecocci P, Polidori MC, Ingegni $\mathrm{T}$ et al: Oxidative damage to DNA in lymphocytes from AD patients. Neurology 1998; 51: $1014-1017$.

27 McKhann G, Drachman D, Folstein M, Katzman R, Price D, Stadlan EM: Clinical diagnosis of Alzheimer's disease: report of the NINCDS-ADRDA Work Group under the auspices of Department of Health and Human Services Task Force on Alzheimer's Disease. Neurology 1984; 34: 939-944.

28 Morrison TB, Weis JJ, Wittwer CT: Quantification of low-copy transcripts by continuous SYBR Green I monitoring during amplification. Biotechniques 1998; 24: 954-958, 960, 962.
29 Ririe KM, Rasmussen RP, Wittwer CT: Product differentiation by analysis of DNA melting curves during the polymerase chain reaction. Anal Biochem 1997; 245: 154-160.

30 Barrientos A, Casademont J, Cardellach F, Estivill X, UrbanoMarquez A, Nunes V: Reduced steady-state levels of mitochondrial RNA and increased mitochondrial DNA amount in human brain with aging. Brain Res Mol Brain Res 1997; 52: 284-289. 